\title{
Transport Aircraft System Identification from Wind Tunnel Data
}

\author{
Patrick C. Murphy* \\ NASA Langley Research Center, Hampton, VA, 23681-2199 \\ Vladislav Klein ${ }^{\dagger}$ \\ National Institute of Aerospace, NASA Langley Research Center, Hampton, VA, 23681-2199
}

\begin{abstract}
Recent studies have been undertaken to investigate and develop aerodynamic models that predict aircraft response in nonlinear unsteady flight regimes for transport configurations. The models retain conventional static and rotary dynamic terms but replace conventional acceleration terms with more general indicial functions. In the Integrated Resilient Aircraft Controls project of the NASA Aviation Safety Program one aspect of the research is to apply these current developments to transport configurations to facilitate development of advanced controls technology. This paper describes initial application of a more general modeling methodology to the NASA Langley Generic Transport Model, a sub-scale flight test vehicle.
\end{abstract}

\section{Nomenclature}

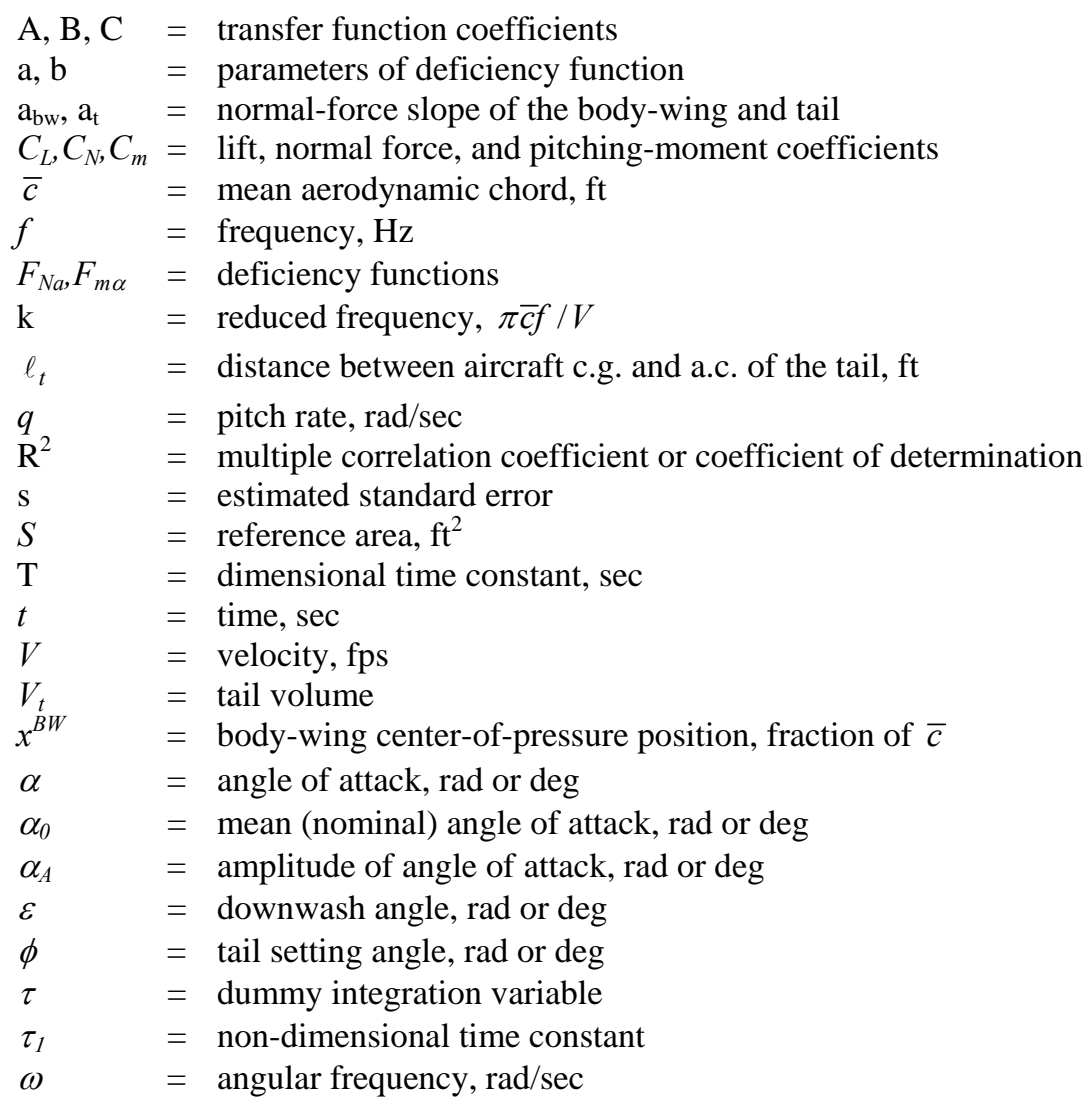

\footnotetext{
* Senior Research Engineer, Dynamic Systems \& Control Branch, Mail Stop 308, Associate Fellow.

${ }^{\dagger}$ Professor Emeritus, Dynamic Systems \& Control Branch, Mail Stop 308, Associate Fellow.
} 


$$
\begin{aligned}
& \text { subscripts } \\
& \begin{array}{ll}
A & =\text { amplitude } \\
b w & =\text { body-wing } \\
t & =\text { tail }
\end{array}
\end{aligned}
$$

Aerodynamic Derivatives

$$
\begin{aligned}
C_{a_{\alpha}} & =\frac{\partial C_{a}}{\partial \alpha} \text { where } a=N \text { or m } \\
C_{a_{q}} & =\frac{\bar{c}}{2 V} \frac{\partial C_{a}}{\partial q} \text { where } a=N \text { or m }
\end{aligned}
$$

abbreviations

$\begin{array}{ll}\text { c.g. } & =\text { center of gravity } \\ \text { a.c. } & =\text { aerodynamic center }\end{array}$

\section{Introduction}

—or conditions that include nonlinear or unsteady flight regimes, modeling and identification of transport aircraft -1 in a classical wing-tail configuration brings new challenges to mathematical model postulation, wind tunnel and flight test experiment design, and aerodynamic parameter estimation.

The problem of model formulation for a wing-tail combination in planar motion was addressed in Refs. 1-3. In all these cases only linear unsteady aerodynamics were considered and no application of the theory was made to experimental data. An extension to a nonlinear unsteady aerodynamic formulation and parameter estimation from wind tunnel data appeared in Ref. 4. In this analysis both steady and oscillatory data were used. The identified model was presented in several graphs comparing measured and estimated data. Unfortunately, no numerical values of estimated parameters and their accuracies were given. For that reason the same data were re-examined in Refs. 5 and 6, addressing mainly measured data inconsistency, low accuracy, non-physical values of parameter estimates, and model adequacy.

In this paper the problem of system identification is considered again. This time, however, new sets of steady and oscillatory data for an airliner are used. These data were selected from extensive data sets produced at NASA Langley during the years from 2000 to 2007. The main distinction between previous and recent sets of data is in the small amplitude oscillatory data. In recent tests, the number and range of test frequencies were increased. The new data allow identification of a model with linear unsteady aerodynamics. In future this model should form a basis for extended models including nonlinear unsteady aerodynamics.

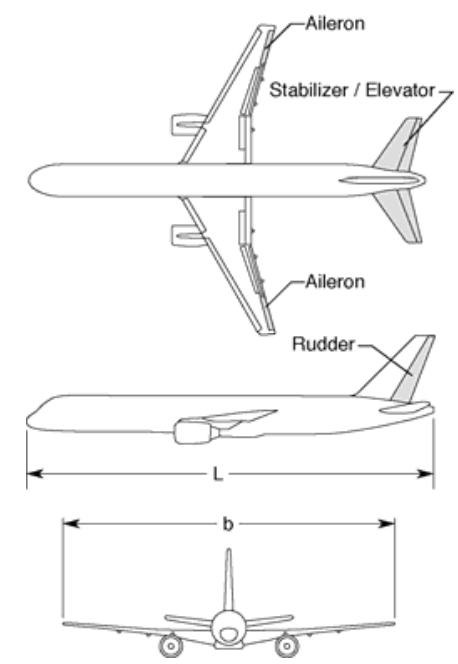

$\mathrm{S}=5.90 \mathrm{ft}^{2}, \bar{c}=0.915 \mathrm{ft}, \mathrm{b}=8.85 \mathrm{ft}$

Figure 1. Model Geometry for NASA experimental sub-scale aircraft.

\section{Measured Data}

In order to obtain an extensive and detailed aerodynamic data base, three steady and dynamic low-speed wind tunnel tests were conducted during 2000-2001, 2003, and 2007, in the NASA Langley 14x22 Wind Tunnel, using a 5.5\% scale model representing a conventional twin-engine commercial airliner. This model has the same mold line specifications as the NASA Langley sub-scale flight test vehicle, referred to as the Generic Transport Model (GTM). The sketch and basic dimensions of GTM are given in Fig. 1. Tests were conducted mostly at a speed of $86 \mathrm{ft} / \mathrm{sec}$, corresponding to a Reynolds Number of $0.5 \times 10^{6}$ based on mean aerodynamic chord. The steady data were obtained for different configurations and an extensive range of angles of attack, sideslip angles, and control surface deflections. The dynamic tests included one degree-of-freedom forced oscillation around the pitch, roll, and yaw axes at different nominal values of the angle of attack, for different frequencies and amplitudes. Commanded oscillations were in the form of single sinusoids. An overview of the first two tests is presented in Refs. 7 and 8.

For the following data analysis, only the normal force and pitching moment coefficient were considered. The steady data were taken from the 2003 experiment. They include the aerodynamic coefficients for body alone (B), body-wing (BW), body-tail (BT), and basic configuration (BWT) at angles of attack from $-10^{\circ}$ to $60^{\circ}$ and for some 
runs to $90^{\circ}$. Data for four configurations are plotted in Figs. 2 and 3. For the BWT case, measurements were made in both directions as the test rig moved up and down the angle-of-attack range. Consequently, at some angles of attack the plot will show two "+" symbols closely spaced.

The oscillatory data were taken from the 2007 experiment for two configurations, BW and BWT, at nominal values of angle of attack in the range $-10^{\circ}$ to $50^{\circ}$, three amplitudes of $5^{\circ}, 10^{\circ}$, and $15^{\circ}$, and typically six frequencies ranging from 0.01 to $0.86 \mathrm{~Hz}$. During the test runs, data were obtained from 7 oscillation cycles for low frequency data, and 14 to 44 cycles for the remaining data. Oscillatory data were sampled at $250 \mathrm{~Hz}$ with $100 \mathrm{~Hz}$ analog filters. The resulting data were further filtered with a $4 \mathrm{~Hz}$ low pass digital filter to remove unwanted frequency content. The filter was run in both directions to ensure no phase error was added to the data. Initial cycles were discarded to avoid any startup transients in the data and five repeated cycles were used for data analysis to ameliorate the effects of measurement noise.

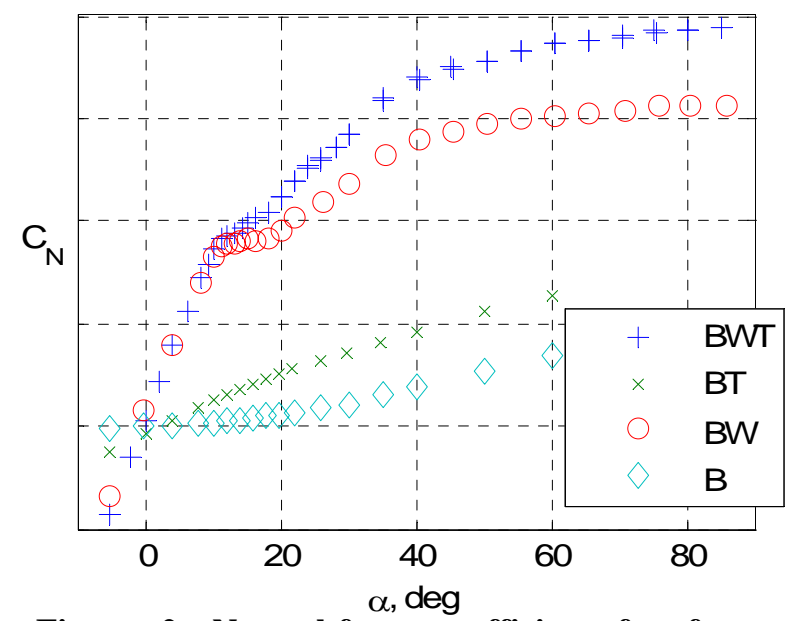

Figure 2. Normal-force coefficient for four model configurations.

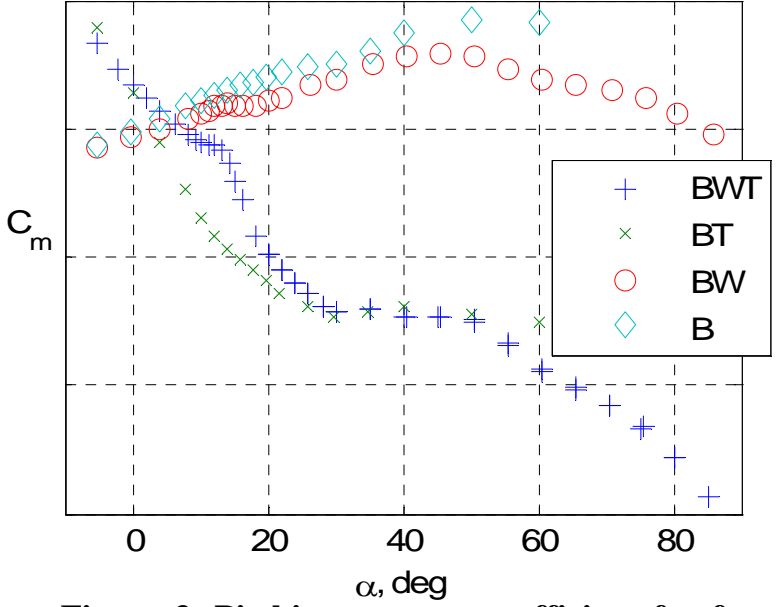

Figure 3. Pitching-moment coefficient for four model configurations.

\section{Analysis of Steady Data}

Selection of mathematical model structure for the analysis of steady data was influenced by the form of measured data (see Figs. 2 and 3) and by experience in modeling, as discussed in Refs. 4 to 6. In this study, model formulation assumes that the aerodynamics of the complete airplane can be formulated as a sum of two contributions, namely, the body-wing and the tail. This assumption is validated later in this section by showing that measured $C_{a}^{B W T}$ is equal to computed $C_{a}^{B W T}=C_{a}^{B W}+C_{a}^{T}$, where subscript $a$ is $N$ or $m$. The aerodynamic coefficients $C_{N}$ and $C_{m}$ can be expressed as

$$
\begin{gathered}
C_{N}(\alpha)=C_{N}^{B W}(\alpha)+\bar{S}_{t} C_{N}^{T}\left(\alpha_{t}\right) \\
C_{m}(\alpha)=C_{m_{0}}^{B W}+C_{N}^{B W}(\alpha) x^{B W}(\alpha)-\bar{\ell}_{t} \bar{S}_{t} C_{N}^{T}\left(\alpha_{t}\right)
\end{gathered}
$$

where

$$
C_{N}^{T}=C_{N}^{B T}-C_{N}^{B}
$$

$$
\alpha_{t}=\alpha+\phi-\varepsilon(\alpha)
$$

Furthermore, $\bar{S}_{t}=S_{t} / S, \bar{\ell}_{t}=\ell_{t} / \bar{c}$, and $x^{B W}$ is the distance between the body-wing aerodynamic center and the aircraft moment reference center as a fraction of mean aerodynamic chord. This distance can be computed from measured BW steady data and using Eq. (2) with the tail terms removed. $C_{N}$ or $C_{m}$ without superscript refers to the complete BWT configuration, $C_{N}^{B W T}$ or $C_{m}^{B W T}$, respectively. The downwash angle at the tail, $\varepsilon(\alpha)$, follows from solving the following two equations for $\alpha_{t}$ at selected $\alpha$. 


$$
\begin{gathered}
C_{m}^{T}(\alpha)=C_{m}^{B T}(\alpha)-C_{m}^{B}(\alpha) \\
C_{m}^{T}(\alpha)=-\bar{\ell}_{t} \bar{S}_{t} C_{N}^{T}\left(\alpha_{t}\right)
\end{gathered}
$$

The variables $x^{B W}(\alpha)$ and $\varepsilon(\alpha)$ are plotted in Figs. 4 and 5.

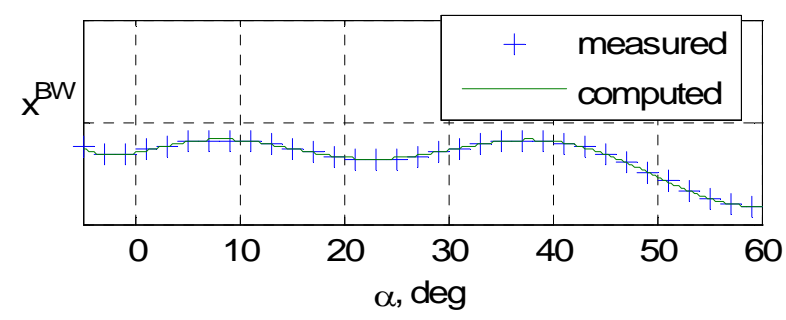

Figure 4. Distance from moment reference center to aerodynamic center.

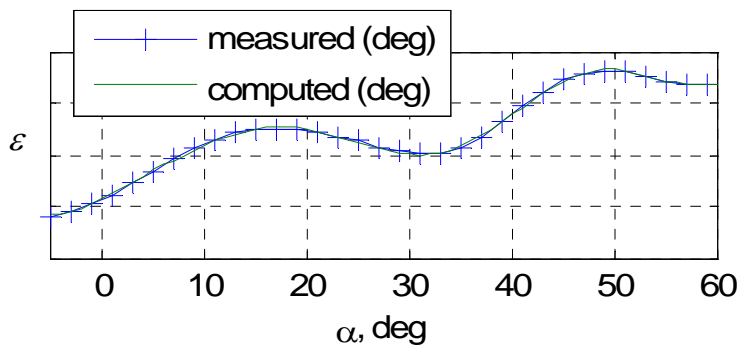

Figure 5. Downwash angle at the tail.

In order to allow steady data computations at any angle of attack, the measured data were fitted by high-order polynomials in $\alpha$. Data compatibility was checked by comparing measured and computed $C_{N}$ and $C_{m}$ using Eqs. (1) to (4). The results are presented in Fig. 6 . The measured and computed coefficients are in very good agreement thus substantiating the use of superposition in forming model equations. The additional check confirmed the equality in Eq. (6) and the validity of $\bar{\ell}_{t}=4.02$. This value is obtained from the model geometry.

Force contributions from the isolated wing and isolated tail can be computed from steady data using Eqs. (3-4) and from the expression

$$
C_{N}^{W}=C_{N}^{B W T}-C_{N}^{B T}
$$

and similarly for moment contributions, $C_{m}^{W}$ and $C_{m}^{T}$. Steady data analysis was completed by considering $C_{L}(\alpha)$, plotted in Fig. 7, since this coefficient shows pre- and post-stall regions more clearly than $C_{N}(\alpha)$.
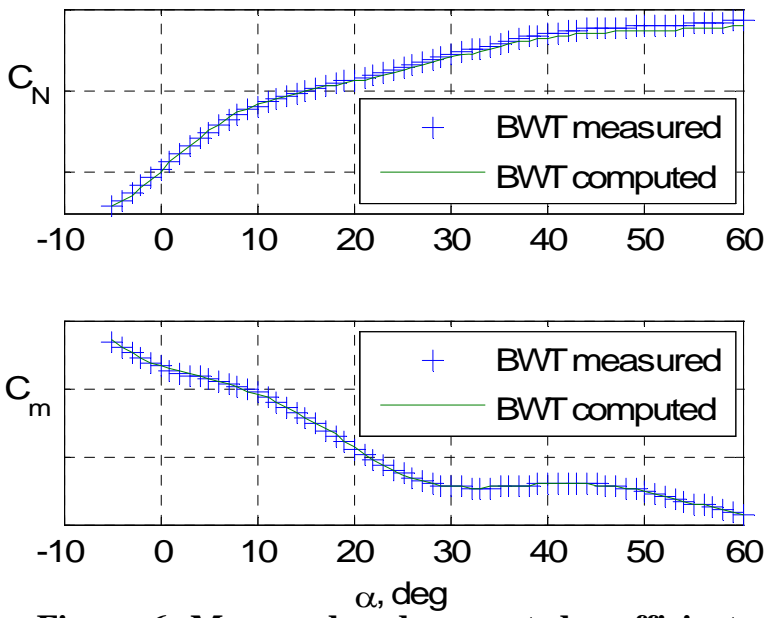

Figure 6. Measured and computed coefficients for complete aircraft.

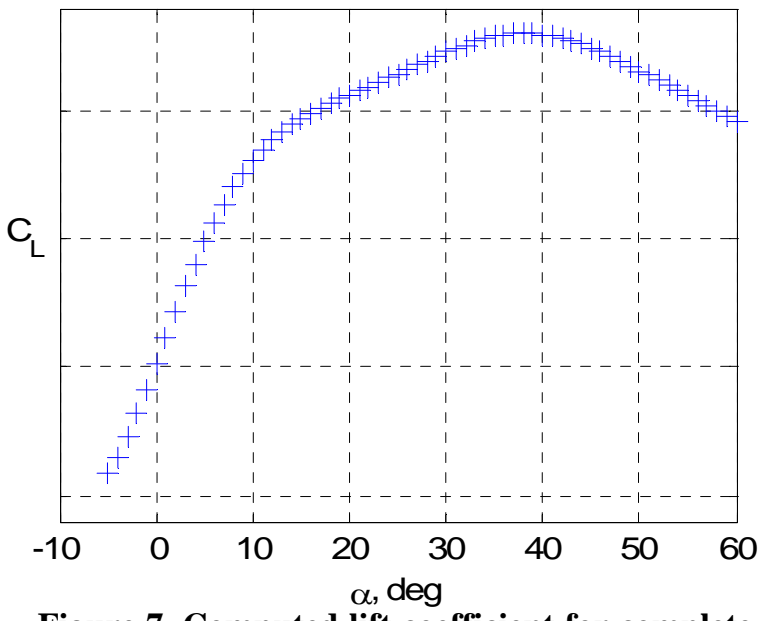

Figure 7. Computed lift coefficient for complete aircraft from normal force and axial force.

\section{Model Formulation with Linear Unsteady Aerodynamics}

Development of linear model equations for an aircraft in one planar motion follows from the results in Refs. 3, 9, and 10. For this study the normal force and pitching moment can be considered as functions of angle of attack and 
pitch rate, i.e., $C_{a}(t)=C_{a}(\alpha(t), q(t))$, where $a=N$ or $m$. Then the aerodynamic model equations can be formulated as

$$
C_{a}(t)=C_{a}(0)+\int_{0}^{t} C_{a \alpha}(t-\tau) \dot{\alpha}(\tau) d \tau+\frac{\bar{c}}{2 V} \int_{0}^{t} C_{a q}(t-\tau) \dot{q}(\tau) d \tau
$$

where $C_{a}(0)$ is the value of the coefficient at initial steady-state conditions, and $C_{a_{\alpha}}(t)$ and $C_{a_{q}}(t)$ are the indicial functions defining the responses in $C_{a}$ to unit step in $\alpha$ and $q$, respectively. Replacing the second indicial function in Eq. (8) by its steady value $C_{a_{q}}(\infty)$, Eq. (8) is simplified as

$$
C_{a}(t)=C_{a}(0)+\frac{\bar{c}}{2 V} C_{L_{q}}(\infty) q(t)+\int_{0}^{t} C_{a \alpha}(t-\tau) \dot{\alpha}(\tau) d \tau
$$

When the deficiency function

$$
F_{a_{\alpha}}(t)=C_{a_{\alpha}}(\infty)-C_{a_{\alpha}}(t)
$$

is substituted into Eq. (9), the equations for the aerodynamic coefficients take the form

$$
\begin{aligned}
& C_{N}(t)=C_{N}(0)+C_{N_{\alpha}}(\infty) \alpha(t)+\frac{\bar{c}}{2 V} C_{N_{q}}(\infty) q(t)-\int_{0}^{t} F_{N_{\alpha}}(t-\tau) \dot{\alpha}(\tau) d \tau \\
& C_{m}(t)=C_{m}(0)+C_{m_{\alpha}}(\infty) \alpha(t)+\frac{\bar{c}}{2 V} C_{m_{q}}(\infty) q(t)-\int_{0}^{t} F_{m_{\alpha}}(t-\tau) \dot{\alpha}(\tau) d \tau
\end{aligned}
$$

where $C_{N_{\alpha}}(\infty), C_{m_{\alpha}}(\infty), C_{N_{q}}(\infty)$, and $C_{m_{q}}(\infty)$ are the partial derivatives with respect to $\alpha$ and $q$, respectively, of the coefficients $C_{N}$ and $C_{m}$ in steady flow conditions.

The indicial functions in Eqs. (11) and (12) include combined responses of the wing, tail, and interference effects between the lifting surfaces. With the assumption of linear aerodynamics the resulting indicial functions are given as a sum of four components. For the indicial function, $C_{N_{\alpha}}(t)$, these components are as follows:

1. the response in the normal force of the wing to a unit step in angle of attack of the wing, while the angle of attack of the tail remains at zero;

2. the response in the normal force of the tail to a unit step in angle of attack of the tail, while the angle of attack of the wing remains at zero;

3. the response in the normal force of the tail to a unit step in angle of attack of the wing, while the angle of attack of the tail remains at zero;

4. response in the normal force of the wing to a unit step in angle of attack of the tail, while the angle of attack of the wing remains at zero.

The first two components represent the response of an isolated wing and isolated tail, respectively. The third component expresses the lift on the tail due to a change in the downwash induced by lift of the wing. It is usually a significant contribution to the resulting pitching moment of an aircraft. For the following data analysis the second and fourth components of the indicial function were assumed to be small relative to total forces or moments and therefore were neglected.

Contributions of the body-wing and tail to coefficients $C_{N}$ and $C_{m}$ are described in the relations

$$
C_{N}=C_{N}^{B W}+\bar{S}_{t} C_{N}^{T}
$$




$$
C_{m}=C_{m_{\text {a.c. }}}+x^{B W} C_{N}^{B W}-V_{t} C_{N}^{T}
$$

where $C_{m_{a . c}}$ is the pitching moment about the aerodynamic center of the body-wing combination, $x^{B W}$ is the distance between the BW aerodynamic center and the aircraft center of gravity or moment reference center, and tail volume, $V_{t}=\bar{\ell}_{t} \bar{S}_{t}$. Combining Eqs. (11-14) yields

$$
\begin{gathered}
C_{N}(t)=C_{N}(0)+C_{N_{\alpha}}(\infty) \alpha(t)+\frac{\bar{c}}{2 V} C_{N_{q}}(\infty) q(t)-\int_{0}^{t}\left[F_{N_{\alpha}}^{B W}(t-\tau)+\bar{S}_{t} F_{N_{\alpha}}^{T}(t-\tau)\right] \dot{\alpha}(\tau) d \tau \\
C_{m}(t)=C_{m_{\text {a.c. }}}+C_{m_{\alpha}}(\infty) \alpha(t)+\frac{\bar{c}}{2 V} C_{m_{q}}(\infty) q(t)-\int_{0}^{t}\left[x^{B W} F_{N_{\alpha}}^{B W}(t-\tau)+V_{t} F_{N_{\alpha}}^{T}(t-\tau)\right] \dot{\alpha}(\tau) d \tau
\end{gathered}
$$

where the deficiency functions are defined as

$$
\begin{gathered}
F_{N_{\alpha}}^{B W}(t)=C_{N_{\alpha}}^{B W}(\infty)-C_{N_{\alpha}}^{B W}(t) \\
F_{N_{\alpha}}^{T}(t)=C_{N_{\alpha}}^{T}(\infty)-C_{N_{\alpha}}^{T}(t)
\end{gathered}
$$

The steady terms in Eq. (15-16) include the aircraft stability derivatives, $C_{N_{\alpha}}, C_{m_{\alpha}}$, $C_{N_{q}}$, and $C_{m_{q}}$. Indicial functions are reduced to these terms under steady flow conditions. The first two derivatives are usually formed as

$$
\begin{gathered}
C_{N_{\alpha}}=a_{b w}+a_{t} \bar{S}_{t}(1-\partial \varepsilon / \partial \alpha) \\
C_{m_{\alpha}}=x^{B W} a_{b w}-a_{t} V_{t}(1-\partial \varepsilon / \partial \alpha)
\end{gathered}
$$

In these equations $a_{b w}=\partial C_{N}^{B W} / \partial \alpha$ and $a_{t}=\partial C_{N}^{T} / \partial \alpha_{t}$ are the normal force slopes of the body-wing and tail, respectively. The derivatives $C_{N_{q}}$ and $C_{m_{q}}$ represent aerodynamic effects that accompany rotation of the aircraft about its lateral axis while $\alpha$ remains constant. Both body-wing and tail are affected by the rotation but the bodywing contribution is usually negligible. Thus considering a contribution of the tail only, the damping parameters are approximated as

$$
\begin{gathered}
C_{N_{q}}=2 a_{t} V_{t} \\
C_{m_{q}}=-2 a_{t} \bar{\ell}_{t} V_{t}
\end{gathered}
$$

For parameter estimation from measured data the form of the deficiency functions in Eqs. (15-16) must be specified. Experience with identification of tailless aircraft indicates that a simple exponential function

$$
F_{N_{\alpha}}(t)=a e^{-b t}
$$

can be a good approximation. In Ref. 3 the model for indicial function $C_{N_{\alpha}}^{T}(t)$ is based on linear aerodynamics and a combination of three effects: (1) change in $C_{N}^{B W}$ due to change in $\alpha$, (2) change in $\varepsilon$ at the tail due to a change in 
$C_{N}^{B W}$ and (3) by a change in $C_{N}^{T}$ due to change in $\varepsilon$. This model, however, is not suitable for parameter estimation due to its complexity.

A simplified form of $C_{N_{\alpha}}^{T}$ was proposed in Ref. 4 assuming that the propagation of the downwash is governed by a first order differential equation

$$
T \dot{\varepsilon}=-\varepsilon+\varepsilon(\infty)
$$

leading to an exponential form of the deficiency function. In this paper a simple form was used for the deficiency function

$$
F_{N_{\alpha}}^{T}(t)=\left\{\begin{array}{ll}
\text { constant } & \text { for } 0 \leq t \leq \Delta t \\
0 & \text { for } t \text { elsewhere }
\end{array}\right\}
$$

This formulation is based on the Cowley-Glauert explanation of the downwash delay ${ }^{10}$. They assumed that a sudden change in the angle of attack of the wing results in a sudden change in the downwash. Downwash from the wing is delayed from reaching the tail by the time $\Delta t=\ell_{t} / V$. Then the contribution of the tail to the normal force ${ }^{11}$ is

$$
\Delta C_{N}^{T}=-a_{t} \bar{S}_{t} \Delta \varepsilon=a_{t} \bar{S}_{t} \frac{\ell_{t}}{V} \frac{\partial \varepsilon}{\partial \alpha} \dot{\alpha}
$$

or further simplifying as

$$
\Delta C_{N}^{T}=2 a_{t} \ell_{t} \bar{S}_{t} \frac{\partial \varepsilon}{\partial \alpha} \frac{\dot{\alpha} \bar{c}}{2 V}=C_{N_{\dot{\alpha}}}^{T} \frac{\dot{\alpha} \bar{c}}{2 V}
$$

and to the pitching moment

$$
\Delta C_{m}^{T}=2 a_{t} \ell_{t} \bar{S}_{t} V_{t} \frac{\partial \varepsilon}{\partial \alpha} \frac{\dot{\alpha} \bar{c}}{2 V}=C_{m_{\dot{\alpha}}}^{T} \frac{\dot{\alpha} \bar{c}}{2 V}
$$

These formulations replace the second integral in Eqs. (15-16) by a constant. From Eqs. (15-16) and Eqs. (26-27) the model equations for the increments in the normal force and the pitching moment take the form

$$
\begin{gathered}
C_{N}(t)=C_{N}(0)+C_{N_{\alpha}} \alpha(t)+\frac{\bar{c}}{2 V} C_{N_{q}}^{\prime} q(t)-a \int_{0}^{t} e^{-b(t-\tau)} \dot{\alpha}(\tau) d \tau \\
C_{m}(t)=C_{m_{a . c .}}+C_{m_{\alpha}} \alpha(t)+\frac{\bar{c}}{2 V} C_{m_{q}}^{\prime} q(t)-a \int_{0}^{t} x^{B W} e^{-b(t-\tau)} \dot{\alpha}(\tau) d \tau
\end{gathered}
$$

where

$$
\begin{aligned}
& C_{N_{q}}^{\prime}=C_{N_{q}}+C_{N_{\dot{\alpha}}}^{T} \\
& C_{m_{q}}^{\prime}=C_{m_{q}}+C_{m_{\dot{\alpha}}}^{T}
\end{aligned}
$$

In model Eqs. (28-29) there are six parameters. Four parameters are the aerodynamic derivatives, $C_{N_{\alpha}}, C_{m_{\alpha}}, C_{N_{q}}^{\prime}$, and $C_{m_{q}}^{\prime}$, and two parameters, $a$ and $b$, characterize the deficiency function. 
By introducing

$$
\eta=\int_{0}^{t} e^{-b(t-\tau)} \dot{\alpha}(\tau) d \tau
$$

and omitting the bias term, the state-space format of Eq. (28) is

$$
\begin{gathered}
\dot{\eta}(t)=-b \eta(t)+\dot{\alpha}(t) \\
C_{N}(t)=C_{N_{\alpha}} \alpha(t)+\frac{\bar{c}}{2 V} C_{N_{q}}^{\prime} q(t)-a \eta(t)
\end{gathered}
$$

Applying the Laplace transform to Eq. (33-34), the transfer function for the normal-force coefficient is obtained as

$$
\frac{C_{N}(s)}{\alpha(s)}=\frac{A s^{2}+B s+C}{s+b}
$$

where $s$ is the Laplace transform variable and

$$
\begin{aligned}
A & =\frac{\bar{c}}{2 V} C_{N_{q}}^{\prime} \\
B & =C_{N_{\alpha}}-a+b \frac{\bar{c}}{2 V} C_{N_{q}}^{\prime} \\
C & =b C_{N_{\alpha}}
\end{aligned}
$$

are the transfer function coefficients.

\section{Model Identification}

As mentioned previously, measured oscillatory data were obtained at different angles of attack, amplitudes, and frequencies for two configurations: the basic model (BWT configuration) and the model without tail (BW configuration). In this study data analysis was primarily focused on data with $\alpha_{A}=10^{\circ}$ because these data formed a set with twenty nominal values of $\alpha$, from $-10^{\circ}$ to $50^{\circ}$, and seven frequencies, from 0.05 to $0.86 \mathrm{~Hz}$. For the set of data with $\alpha_{A}=5^{\circ}$, the number of frequencies was reduced to six and the number of $\alpha_{0}$ to twelve. Data with $\alpha_{A}=15^{\circ}$ were not analyzed for this paper but will be used for future research in nonlinear modeling. Model identification for this study includes an assessment of model linearity and its dependence on angle of attack, amplitude, and frequency. Model identification is completed by parameter estimation and model validation.

\section{A. Harmonic Analysis}

A method of harmonic analysis ${ }^{12}$ was applied to measured aerodynamic force and moment coefficients to allow estimation of in-phase and out-of-phase coefficients. Five cycles of forced-oscillation data were analyzed for each frequency. A mathematical model was postulated as

$$
C_{a}(t)=A_{0}+\sum_{j=1}^{m} A_{j} \cos (j \omega t)+\sum_{j=1}^{m} B_{j} \sin (j \omega t) \quad a=N \text { or } m
$$

where $\omega=2 \pi f$ and $A_{0}, A_{1}, B_{1}, \ldots, A_{m}, B_{m}$ are the Fourier coefficients. The analysis provided estimates of these coefficients, their standard errors, and the coefficient of determination, $R^{2}$. This coefficient indicates how much information in the data is explained by the model ${ }^{13}$. For a model with linear aerodynamics, the in-phase and out-ofphase components of $C_{a}(t)$, i.e., $\bar{C}_{a_{\alpha}}$ and $\bar{C}_{a_{q}}$, can be expressed in terms of coefficients $A_{l}$ and $B_{l}$ as 


$$
\begin{array}{ll}
\bar{C}_{a_{\alpha}}=\frac{B_{1}}{\alpha_{A}}=C_{a_{\alpha}}-k^{2} C_{a_{\dot{q}}} & a=N \text { or } m \\
\bar{C}_{a_{q}}=\frac{A_{1}}{k \alpha_{A}}=C_{a_{q}}+C_{a_{\dot{\alpha}}} & a=N \text { or } m
\end{array}
$$

where $k=\pi \bar{c} f / V$ is the reduced frequency. Also shown in Eqs. (38-39), are the commonly expressed relationships of these coefficients to the steady flow damping terms and acceleration terms.

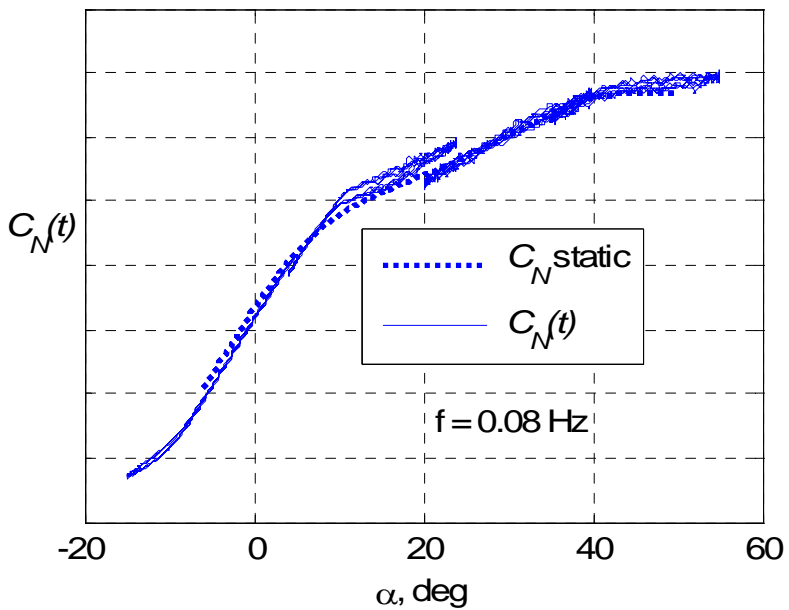

Figure 8. Normal-force coefficient from static and oscillatory data, $f=0.08 \mathrm{~Hz}, \alpha_{A}=10^{\circ}$.

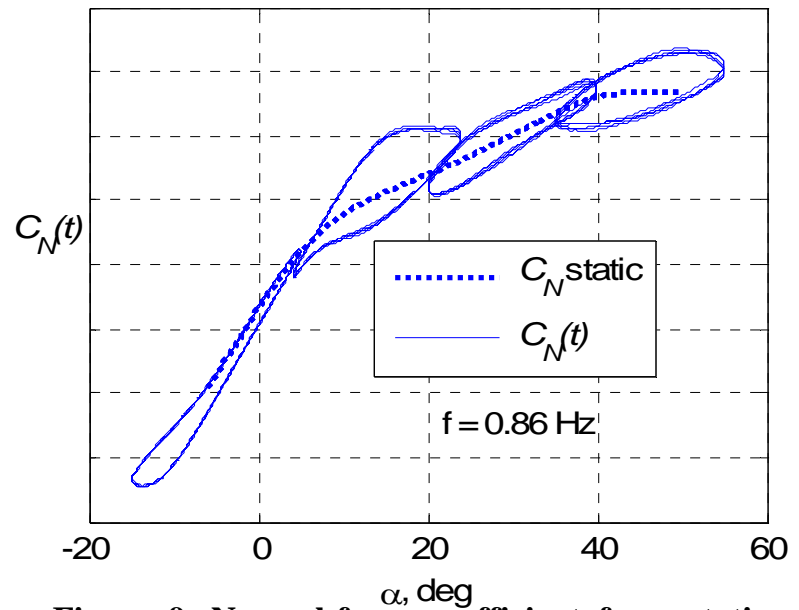

Figure 9. Normal-force coefficient from static and oscillatory data, $f=0.86 \mathrm{~Hz}, \alpha_{A}=10^{\circ}$.

Examples of the measured normal-force coefficient from steady and oscillatory tests are shown in Fig. 8 and 9. Five cycles of oscillatory data are presented for $\alpha_{0}$ at $-5^{\circ}, 14^{\circ}, 30^{\circ}$, and $45^{\circ}$, and for $f=0.08$ and $0.86 \mathrm{~Hz}$. Fig. 9 highlights the effect of increased steady-flow damping at higher $f$ and $\alpha_{0}>10^{\circ}$. The departure of the oscillatory data from a regular ellipse indicates a departure from linear aerodynamics.

The estimated parameters $\bar{C}_{a_{\alpha}}$ and $\bar{C}_{a_{q}}$ for $a=N$ or $m$, are presented in Fig. 10 and 11 for BWT configurations and $\alpha_{A}=10^{\circ}$. The estimates of $\bar{C}_{N_{\alpha}}$ and $\bar{C}_{m_{\alpha}}$ change very little with frequency and their values agree closely with derivatives $C_{N_{\alpha}}$ and $C_{m_{\alpha}}$ estimated from steady measurements. On the other hand, parameters $\bar{C}_{N_{q}}$ and $\bar{C}_{m_{q}}$ exhibited strong frequency dependence for $f<0.43 \mathrm{~Hz}$ and $10^{\circ}<\alpha_{0}<50^{\circ}$. Both figures also include estimates of $\mathrm{R}^{2}$. These values indicate that a linear aerodynamic model may be adequate for $-10^{\circ}<\alpha_{0}<30^{\circ}$. Figure 12 includes only the out-of-phase components of $C_{N}$ and $C_{m}$ for BW configuration and $\alpha_{A}=10^{\circ}$. Figs. 10-12, show the contribution of the tail to aircraft damping.

In order to explain the variation of the in-phase and out-of-phase components with frequency these components were expressed in terms of deficiency functions ${ }^{14,15}$, as in Eq. (23). In Refs. 14-15, the model for these components has the form

$$
\begin{gathered}
\bar{C}_{a_{\alpha}}=C_{a_{\alpha}}-a \frac{\tau_{1}^{2} k^{2}}{1+\tau_{1}^{2} k^{2}} \\
\bar{C}_{a_{q}}=C_{a_{q}}-a \frac{\tau_{1}}{1+\tau_{1}^{2} k^{2}}
\end{gathered}
$$


where subscript $a=N$ or $m$. The second terms on the right side of Eqs. (40-41) can be considered as an unsteady counterpart of $k^{2} \bar{C}_{a_{\dot{q}}}$ and $\bar{C}_{a_{\dot{\alpha}}}$. For the model Eqs. (40-41), there are four unknown parameters $\left(C_{a_{\alpha}}, C_{a_{q}}, a, \tau_{1}\right)$ which can be estimated from $\bar{C}_{a_{\alpha}}$ and $\bar{C}_{a_{q}}$.
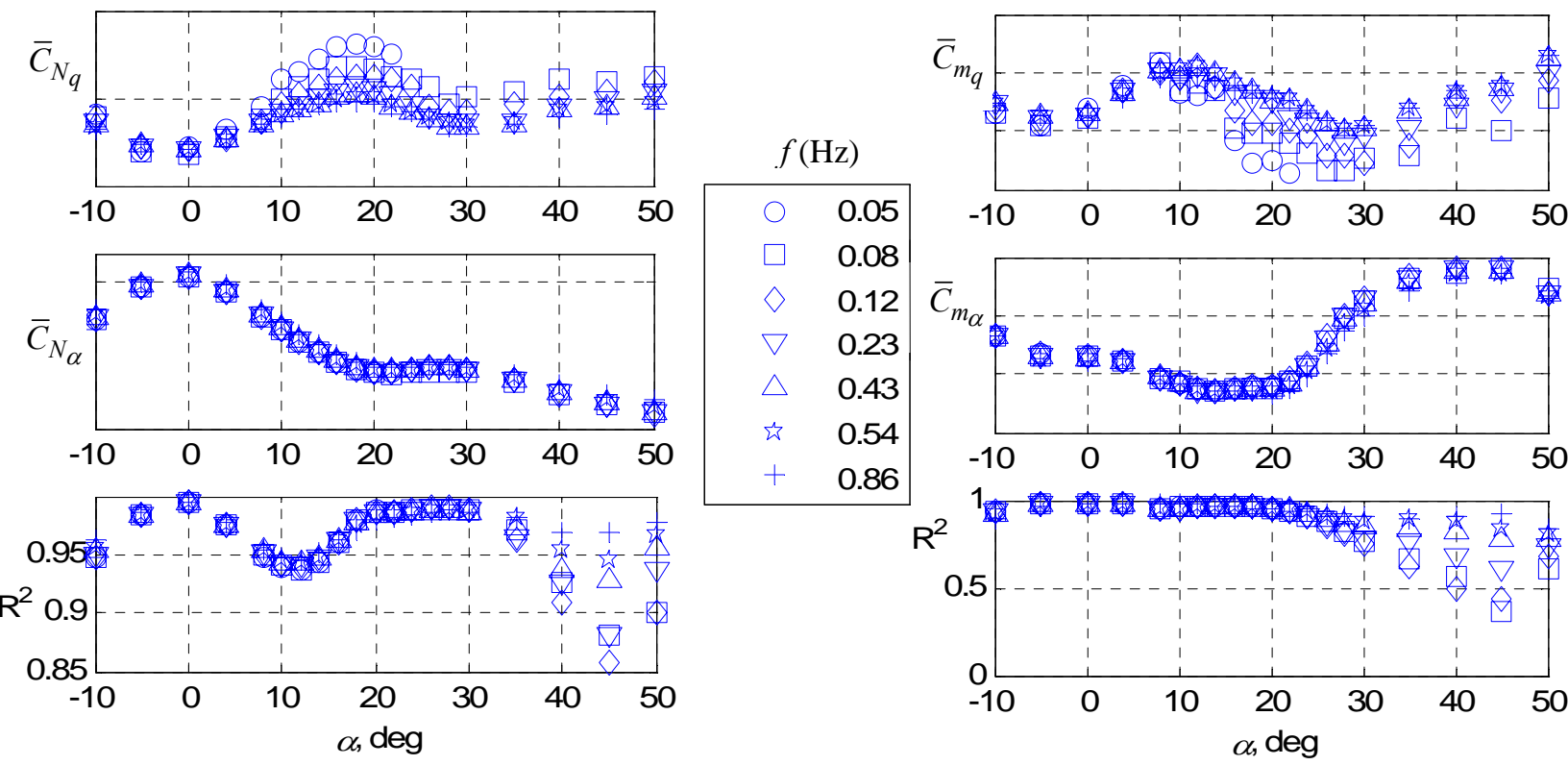

Figure 10. Harmonic analysis for normal-force coefficient, BWT configuration, $\alpha_{A}=10^{\circ}$.

Figure 11. Harmonic analysis for pitch-moment coefficient, BWT configuration, $\alpha_{A}=10^{\circ}$.

To demonstrate the effect of amplitude on the normal-force coefficient, a plot of five cycles of $C_{N}(t)$ vs $\alpha$ is provided in Fig. 13 for $\alpha_{A}=5^{\circ}, f=0.86 \mathrm{~Hz}$, and $\alpha_{0}$ at $0^{\circ}, 10^{\circ}, 20^{\circ}$, and $30^{\circ}$. As in the $\alpha_{A}=10^{\circ}$ case, the steady values of $C_{N}$ were included for comparison. The small amplitude oscillatory data forms shapes much closer to a regular ellipse, except near the stall break. This supports an assumption of linear aerodynamic responses for these conditions.
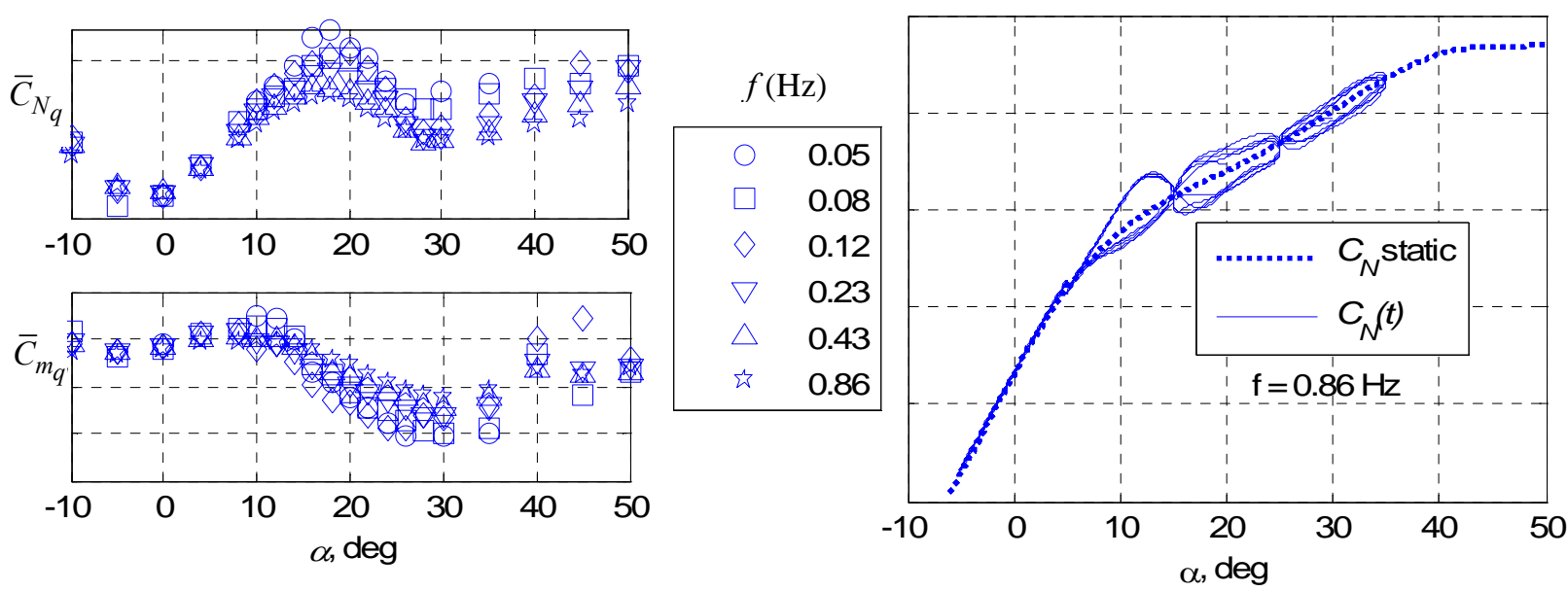

Figure 12. Out-of-phase component of normal force and pitching moment, $\mathrm{BW}, \alpha_{\mathrm{A}}=10^{\circ}$.

Figure 13. Normal-force coefficient from static and oscillatory data, $f=0.86 \mathrm{~Hz}, \mathrm{BW}, \alpha_{\mathrm{A}}=5^{\circ}$.

Figs. 14-15 contain out-of-phase components, $\bar{C}_{N_{q}}$ and $\bar{C}_{m_{q}}$, for both BWT and BW configurations with $\alpha_{A}=$ $5^{\circ}$. Some differences are visible when comparing these results with the estimates at $\alpha_{A}=10^{\circ}$. The main difference is 
the effect of frequency on $\bar{C}_{m_{q}}$ for $10^{\circ}<\alpha_{0}<30^{\circ}$. Explanation of this effect, amplitude effects, and other differences will be covered in future research.
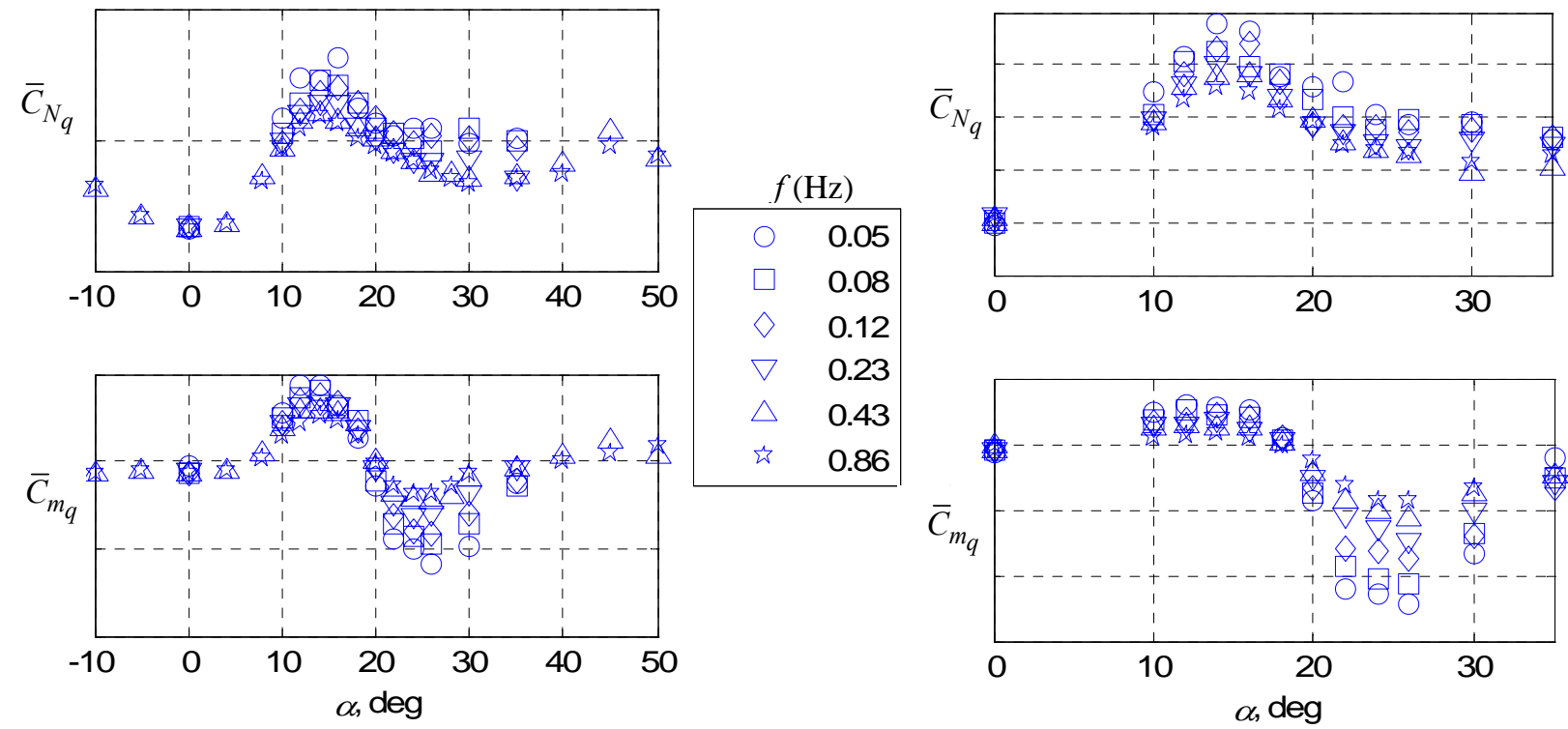

Figure 14. Out-of-phase components for normalforce and pitching moment, BWT, $\alpha_{A}=5^{\circ}$.

Figure 15. Out-of-phase components for normalforce and pitching moement, BW, $\alpha_{A}=5^{\circ}$.

If the principle of superposition applies to forced-oscillation data, then the damping parameter of the tail alone can be computed as

$$
\bar{C}_{N_{q}}^{T}=\bar{C}_{N_{q}}^{B W T}-\bar{C}_{N_{q}}^{B W}
$$

Parameter $\bar{C}_{N_{q}}^{T}$ is plotted in Fig. 16 against frequency and four values of $\alpha_{0}$. This plot indicates little frequency effect for $f>0.2 \mathrm{~Hz}$, however, for lower frequencies the value is rapidly increased. Similar behavior was observed for parameter $\bar{C}_{m_{q}}^{T}$. These changes and the effect of introducing deficiency function $F_{N_{\alpha}}^{T}$ are not explicitly included in the current model formulation, see Eqs. (28-29), and have not been studied.

\section{B. Parameter Estimation}

For estimation of aerodynamic derivatives and indicial (or deficiency) function parameters, two approaches are used: 1) a two step linear regression of Ref. 12 or 2) a variation of an output error method explained in Ref. 13 and implemented in Ref. 16. The

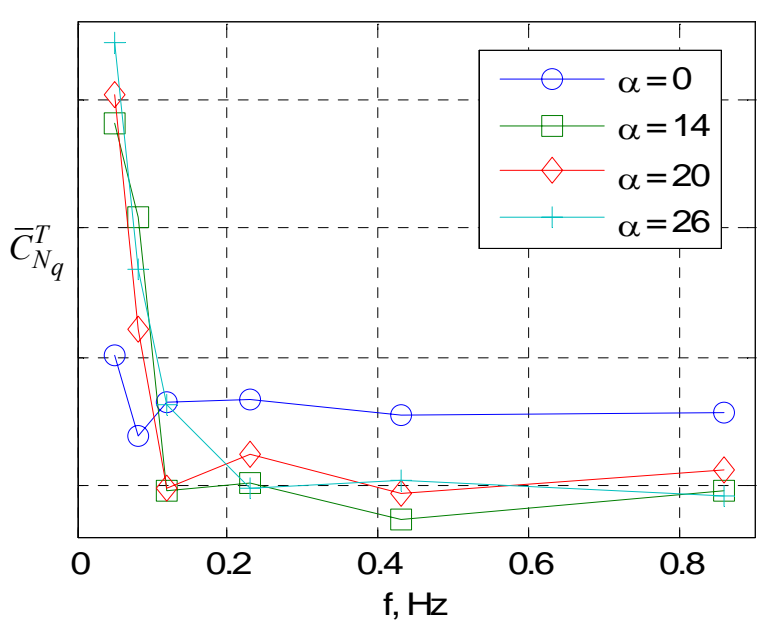

Figure 16. Out-of-phase components for tail alone, $\alpha_{A}=10^{\circ}$. two-step linear regression is based on model Eqs. (40) and (41). This method estimates $\tau_{l}$ in the first step as the only unknown parameter. Then, in the second step, $\tau_{1}$ is treated as a known parameter and the remaining three parameters, $C_{N_{\alpha}}, C_{N_{q}}$ and $a$, are estimated. This method works very well provided that a distinct linear relationship exists between $\bar{C}_{N_{\alpha}}$ components and $\bar{C}_{N_{q}}$ for different frequencies at a given $\alpha_{0}$. This is not the case for the present experiment as follows from the lack of frequency dependence in $\bar{C}_{a_{\alpha}}$, shown Figs. 10 and 11 . In this 
study an output error based method ${ }^{9}$ was used for estimation of parameters in state-space model Eqs. (33) and (34) that allowed use of the time-domain output error algorithm ${ }^{16}$ to utilize harmonic data. In this case, inputs, $\alpha(t)$,

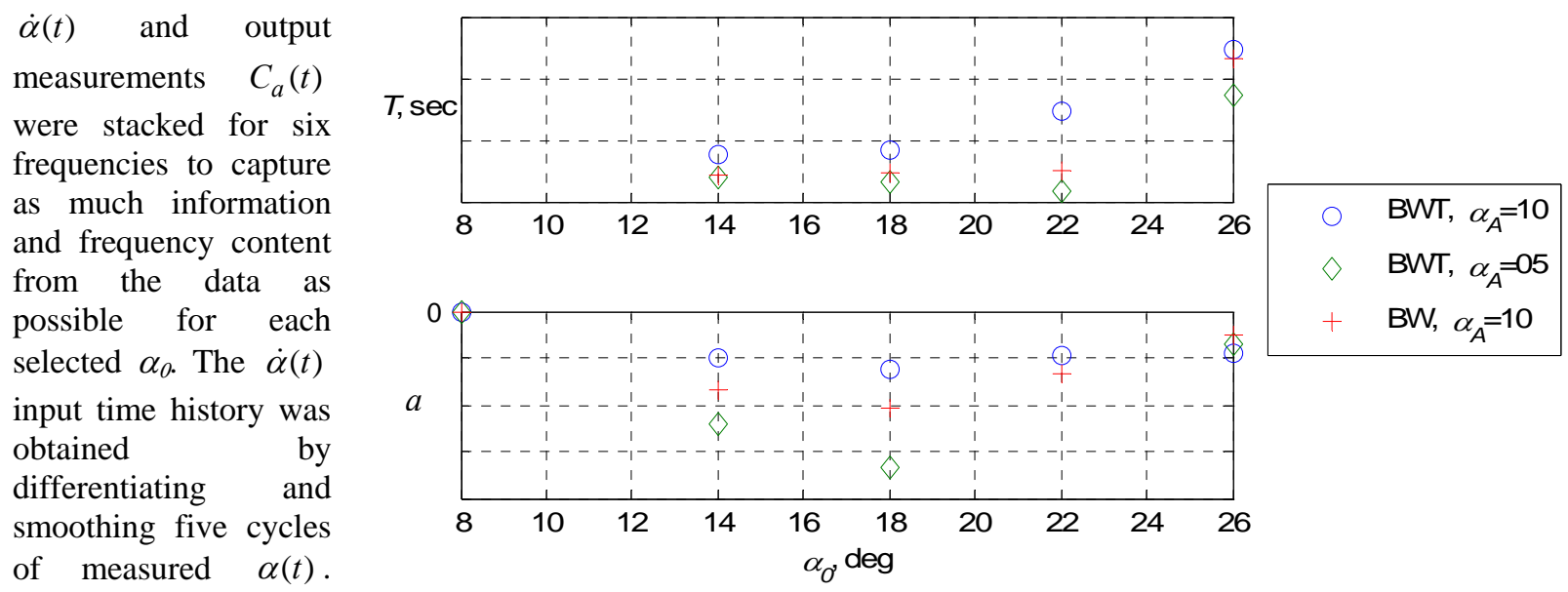

Parameter estimation

Figure 17. Variation of unsteady term parameters, $t$ and a, with frequency. results were obtained at four values of $\alpha_{0}$, two configurations, BWT and BW, and two amplitudes, $\alpha_{A}=5^{\circ}$ and $10^{\circ}$. The results show that estimates of $C_{N_{\alpha}}$ were very close to $\bar{C}_{N_{\alpha}}$ from harmonic analysis and $C_{N_{\alpha}}$ from steady measurements. The average standard error of estimated parameters $C_{N_{\alpha}}$ was 0.0025 . Estimates of $C_{N_{q}}$ with an average standard error 0.68 came close to $\bar{C}_{N_{q}}$ for the highest frequency covered by the experiment. Estimates of parameter $b$ were converted into estimates of the time constant, $T=1 / b$. This parameter is plotted in Fig. 17 against $\alpha$ for two configurations and two amplitudes. The results indicate a gradual increase of time constant with $\alpha$. For better clarity, parameter error bars were not included in Fig. 17. Variation of estimated parameter $a$ for different configurations and angles of attack is also shown in Fig. 17. Trend in the data follows an expected variation from zero (in low $\alpha$ regions without unsteady effect) and back toward zero for higher $\alpha$ where the steady model is adequate.

Representative contributions from each term in the $C_{N}(t)$ output equation are shown in Fig. 18 for a selected frequency. The main contributors are the static and steady flow damping term. The unsteady term provides a relatively small component to the total response for each frequency and each of the four $\alpha_{0}$ cases considered.

\section{Model Validation}

For validation of the model as a predictor, a comparison of measured and predicted data was used. Fig. 19 show the variation of $C_{N}$ with $\alpha$, at $f=0.86 \mathrm{~Hz}$, two nominal values of $\alpha_{0}\left(14^{\circ}, 26^{\circ}\right)$ and $\alpha_{A}=10^{\circ}$. In both cases the shape of the predicted data forms a regular ellipse reflecting the underlying linear aerodynamic model structure. The measured $C_{N}$ shows some deviation from a regular ellipse, or linear behavior, in the pre-stall and stall region. At $\alpha_{0}$ $=26^{\circ}$, the behavior of the data supports using a linear model. Considering the data for $\alpha_{A}=5^{\circ}$, the predicted data are very close to the measured data for both $\alpha_{0}$ conditions. However, the shape of measured data for $\alpha_{0}=14 \operatorname{deg}$ is still somewhat affected by the aerodynamics of the stall region. 

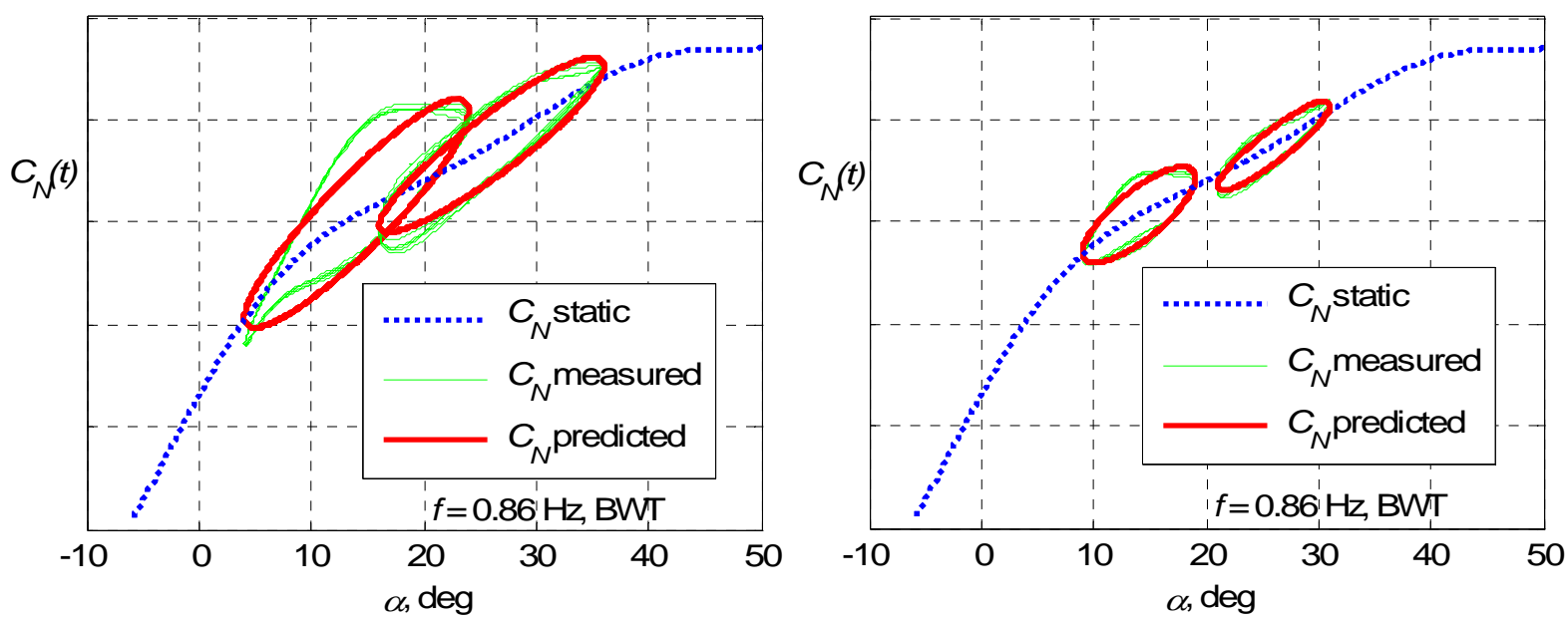

Figure 19. Comparison of measured and predicted Figure 20. Comparison of measured and predicted normal-force coefficient, BWT, $\mathrm{f}=\mathbf{0 . 8 6} \mathrm{Hz}, \alpha_{A}=10^{\circ}$. normal-force coefficient, BWT, $\mathrm{f}=\mathbf{0 . 8 6} \mathrm{Hz}, \alpha_{A}=5^{\circ}$.

\section{Concluding Remarks}

This paper presents aircraft model identification from wind tunnel data of a transport aircraft and its components: body-wing-tail, body-wing, and tail. The measured data include results from steady and oscillatory tests over an extended range of angle of attack, frequency, and two amplitudes.

Using the steady data it was shown that the measured normal force and pitching moment for a complete model are identical to those obtained as a sum of body-wing and tail contributions. Accepting a superposition principle, it was possible to estimate the distance from a moment reference center and the body-wing aerodynamic center, as well as the downwash angle at the tail. It was also possible to prove a consistency between normal-force and pitching-moment data, and to compute the normal force of isolated wing and isolated tail.

Static stability derivatives, $C_{a_{\alpha}}$, estimated from oscillatory data were consistent with calculated values using high-order polynomials in $\alpha$ fit to static measurements.

The analysis of oscillatory data was preceded by formulation of a mathematical model for aircraft in planar motion with one degree of freedom and with linear unsteady aerodynamics. This model, in the state space form, was later used for parameter estimation. The data for this approach were obtained from measurements with two configurations (complete model and model without tail), seven frequencies, and two amplitudes of $5^{\circ}$ and $10^{\circ}$.

For harmonic analysis of the data, a mathematical model was defined by equations of motion representing a steady harmonic solution. Model parameters included the in-phase and out-of phase components of normal force and pitching moment and two parameters of an indicial function: time constant, $T$, and a gain, $a$, characterizing the level of contribution from the unsteady aerodynamic terms. Estimates were completed by including parameter standard errors and the coefficient of determination for normal force and pitching moment models.

Harmonic analysis indicated that a linear unsteady model is adequate for angles of attack from $-10^{\circ}$ to $30^{\circ}$ for both amplitudes, although some limited nonlinearities present in the pre- and post-stall regions slightly reduced the effectiveness of the linear model. The in-phase components are very close to results from steady data and the out-ofphase components at high frequencies are close to steady damping term. The indicial-term time constant is increasing with an increase of angle of attack. The second parameter of the indicial function correctly indicated the region where the unsteady effect is close to zero.

The resulting model was validated by its prediction capabilities. Measured and predicted normal-force coefficients at different nominal values of the angle of attack and amplitudes were compared. As expected the linear unsteady model with parameters estimated from low amplitude data was a better predictor than the model with parameters from experiment with higher amplitude.

Future work will expand model identification of the NASA Generic Transport Model for lateral-directional axes and investigate higher amplitude and higher $\alpha$ regions where the nonlinear unsteady model is required. 


\section{Acknowledgments}

The authors would like to thank Mr. Gautam Shah from NASA Langley Research Center for providing the experimental data and for numerous discussions on data handling.

\section{References}

${ }^{1}$ Jones, Robert T. and Fehlner, Leo F., “Transient Effects of the Wing Wake on the Horizontal Tail,” NACA TN-771, 1940.

${ }^{2}$ Tobak, Murray, "On the Use of Indicial Function Concept in the Analysis of Unsteady Motions of Wings and Wing-Tail Combinations, NACA Rep. 1188, 1954.

${ }^{3}$ Klein, Vladislav, "Modeling of Longitudinal Unsteady Aerodynamics of a Wing-Tail Combination,” NASA CR-1999209547, September, 1999.

${ }^{4}$ Khrabrov, A., Vinogradov, Yu, and Abromov, N., "Mathematical Modeling of Aircraft Unsteady Aerodynamics at High Incidence with Account of Wing-Tail Interaction,” AIAA Atmospheric Flight Mechanics Conference, AIAA 2004-5278, AIAA Washington, DC, 2004.

${ }^{5}$ Murphy, Patrick C. and Klein, Vladislav, "Estimation of Longitudinal Unsteady Aerodynamics of a Wing-Tail Combination from Wind Tunnel Data,” AIAA Atmospheric Flight Mechanics Conference, AIAA 2006-6154, AIAA Washington, DC, 2006.

${ }^{6}$ Murphy, Patrick C. and Klein, Vladislav, "On Problems Associated with Modeling Wing-Tail Configurations from Wind Tunnel Data,” AIAA Atmospheric Flight Mechanics Conference, AIAA 2007-6722, AIAA Washington, DC, 2007.

${ }^{7}$ Shah, Gautam H., et. al, "Wind-tunnel Investigation of Commercial Transport Aircraft Aerodynamics at Extreme Flight Conditions,” SAE Technical Paper 2002-01-2912, 2002.

${ }^{8}$ Brandon, Jay M., et. al, "Comparison of Rolling Moment Characteristics During Roll Oscillations for a Low and High Aspect Ratio Configuration,” AIAA Atmospheric Flight Mechanics Conference, AIAA 2004-5273, AIAA Washington, DC, 2004.

${ }^{9}$ Klein, Vladislav and Murphy, Patrick C., "Estimation of Aircraft Nonlinear Unsteady Parameters From Wind Tunnel Data," NASA TM-1998-208969, December, 1998.

${ }^{10}$ Cowley, W. L. and Glauert, H., "The Effect of the Lag of the Downwash on the Longitudinal Stability of an Airplane and on the Rotary Derivative Mq,”. R. \& M. 718, February, 1921.

${ }^{11}$ Etkin, Bernard, “Dynamics of Atmospheric Flight,” John Wiley \& Sons, Inc., New York, 1972.

${ }^{12}$ Klein, Vladislav, Murphy, Patrick C., and Szyba, Nathan M., “Analysis of Wind Tunnel Oscillatory Data of the F-16XL Aircraft,” NASA TM-2004-213246, August, 2004.

${ }^{13}$ Klein, Vladislav and Morelli, Eugene A., “Aircraft System Identification: Theory and Practice,” American Institute of Aeronatutics and Astronautics, Inc., Education Series, 2006.

${ }^{14}$ Klein, Vladislav and Norderer, Keith D., "Modeling of Aircraft Unsteady Aerodynamic Characteristics. Part I - Postulated Models,” NASA TM 109120, 1994.

${ }^{15}$ Murphy, Patrick C. and Klein, Vladislav, "Validation of Methodology for Estimating Aircraft Unsteady Aerodynamic Parameters From Dynamic Wind Tunnel Tests,” AIAA Atmospheric Flight Mechanics Conference, AIAA 2003-5397, 2003, AIAA Washington, DC, 2007.

${ }^{16}$ Morelli, E.A., "System Identification Programs for Aircraft (SIDPAC)," AIAA-2002-4704, AIAA Atmospheric Flight Mechanics Conference, Monterey, CA, August 2002. 